\title{
Quorum Sensing N-acyl Homoserine Lactones-SdiA Suppresses Escherichia coli-Pseudomonas aeruginosa Conjugation through Inhibiting tral Expression
}

\author{
Yang $\mathrm{Lu}^{1,2 \dagger}$, Jianming Zeng ${ }^{1 \dagger}$, Binning $\mathrm{Wu}^{3}$, Shunmei $\mathrm{E}^{1}$, Lina Wang ${ }^{1}$, Renxin Cai ${ }^{1}$, \\ Ni Zhang ${ }^{4}$, Youqiang $\mathrm{Li}^{1}$, Xianzhang Huang ${ }^{1,2}$, Bin Huang ${ }^{5 *}$ and Cha Chen ${ }^{1 *}$ \\ 1 Department of Laboratory Medicine, The Second Affiliated Hospital of Guangzhou University of Chinese Medicine, \\ Guangzhou, China, ${ }^{2}$ Postdoctoral Mobile Station, Guangzhou University of Chinese Medicine, Guangzhou, China, ${ }^{3}$ The \\ Second Clinical College, Guangzhou University of Chinese Medicine, Guangzhou, China, ${ }^{4}$ Clinical Microbiology Laboratory, \\ Guangdong Academy of Medical Science and Guangdong General Hospital, Guangzhou, China, ${ }^{5}$ Department of Laboratory \\ Medicine, The First Affiliated Hospital of Sun Yat-sen University, Guangzhou, China
}

OPEN ACCESS

Edited by:

Ghassan M. Matar,

American University of Beirut,

Lebanon

Reviewed by:

Eric Déziel,

Institut National de la Recherche

Scientifique, Canada

Shouguang Jin

University of Florida, USA

*Correspondence:

Bin Huang

huangb3@mail.sysu.edu.cn

Cha Chen

chencha906@163.com

${ }^{t}$ These authors have contributed equally to this work.

Received: 19 October 2016 Accepted: 05 January 2017 Published: 20 January 2017

Citation:

Lu Y, Zeng J, Wu B, ES, Wang L, Cai R, Zhang N, Li Y, Huang $X$, Huang B and Chen C (2017) Quorum

Sensing N-acyl Homoserine

Lactones-SdiA Suppresses Escherichia coli-Pseudomonas aeruginosa Conjugation through Inhibiting tral Expression. Front. Cell. Infect. Microbiol. 7:7. doi: 10.3389/fcimb.2017.00007
Conjugation is a key mechanism for horizontal gene transfer and plays an important role in bacterial evolution, especially with respect to antibiotic resistance. However, little is known about the role of donor and recipient cells in regulation of conjugation.

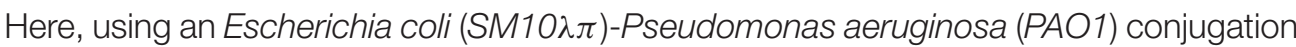
model, we demonstrated that deficiency of lasl/rhll, genes associated with generation of the quorum sensing signals $\mathrm{N}$-acyl homoserine lactones (AHLs) in PAO1, or deletion of the AHLs receptor SdiA in the donor $S M 10 \lambda \pi$ both facilitated conjugation. When using another AHLs-non-producing E. coli strain EC600 as recipient cells, deficiency of sdiA in donor $S M 10 \lambda \pi$ hardly affect the conjugation. More importantly, in the presence

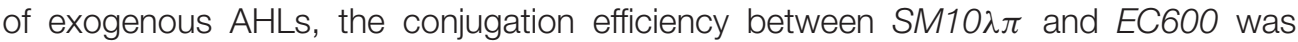

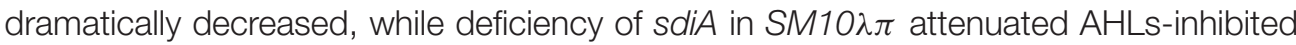
conjugation. These data suggest the conjugation suppression function of AHLs-SdiA chemical signaling. Further bioinformatics analysis, $\beta$-galactosidase reporter system and electrophoretic mobility shift assays characterized the binding site of SdiA on the promoter region of tral gene. Furthermore, deletion of lasl/rhll or sdiA promoted tral mRNA expression in SM10 $\pi \pi$ and PAO1 co-culture system, which was abrogated by AHLs. Collectively, our results provide new insight into an important contribution of quorum sensing system AHLs-SdiA to the networks that regulate conjugation.

Keywords: conjugation, $\mathrm{N}$-acyl homoserine lactones, $P$. aeruginosa, SdiA, antibiotic resistance

\section{INTRODUCTION}

The acquisition of antibiotic resistance by pathogenic microorganisms is a threat to public health worldwide. Horizontal gene transfer, especially conjugative transfer of plasmids that carry resistance genes, is the primary cause of bacterial antibiotic resistance and-on the larger scalebacterial evolution (Zatyka and Thomas, 1998; Arthur et al., 2011). The self-transmissible plasmids, 
such as the well-studied fertility F-plasmids and IncP plasmid RP4 (also known as RK2), generally present a mobilization (MOB) region which includes the origin of transfer (oriT) and the relaxase gene. The relaxase, identified as being TraI in RP4, initiates conjugation by cleaving the oriT in a site- and strandspecific manner (Carballeira et al., 2014). Other plasmids, termed mobilizable, are incapable of initiating conjugation, but can transfer by using the conjugative apparatus of another plasmid (Zatyka and Thomas, 1998). Mobilizable plasmids are more frequently found in natural environment; therefore, replication and mobilization can be considered as important mechanisms that influence plasmid promiscuity (Fernández-López et al., 2014).

Many Gram-negative bacteria utilize $N$-acyl homoserine lactones (AHLs) as signal molecules to enable individual bacteria to coordinate their behavior in populations; such quorum sensing (QS) enables bacteria to not only sense members of their own species but other species as well (Smith et al., 2011). The essential constituents of QS include a signal producer, or synthase, and a cognate transcriptional regulator that responds to the accumulated signal molecules (Bassler and Losick, 2006). The opportunistic animal and plant pathogen Pseudomonas aeruginosa possesses one of the best-studied models of QS, and two different AHL systems, las and rhl, have been identified (Wagner et al., 2003). In the las QS system, the lasI gene product directs formation of the diffusible extracellular signal $N$-(3-oxododecanoyl)-L-HSL (3-oxo-C12-HSL), which interacts with LasR to activate a number of virulence genes including the LasA and LasB elastases, exotoxinA, and alkaline protease (Toder et al., 1991; Gambello et al., 1993; Jones et al., 1993; Passador et al., 1993). In the $r h l$ system, the rhlI gene product catalyzes the synthesis of $N$-butanoyl-L-HSL (C4-HSL). This diffusible signaling molecule, together with RhlR, activates directly some virulence genes like those encoding rhamnolipids and pyocyanin, and represses those genes responsible for assembly and function of the type III secretion system (Bleves et al., 2005; Jimenez et al., 2012). Besides the fact that the las and rhl systems are hierarchically connected, both $r h l R$ and $r h l I$ are positively regulated by the las system (Wagner et al., 2003). The roles of QS in diverse biological processes, such as virulence, biofilm formation and metabolism in $P$. aeruginosa have attracted research attention (Pearson et al., 1994; Hassett et al., 1999; Whiteley et al., 1999; García-Contreras, 2016). However, as the cell-to-cell communication system, the influence of QS on interspecies conjugation remains largely unknown.

Some organisms, such as Escherichia, Klebsiella, Salmonella and Shigella lack AHL synthase and therefore do not produce AHLs; however, they possess a LuxR homolog known as SdiA that can bind AHLs produced by other microorganisms and affect gene expression(Smith and Ahmer, 2003; Yao et al., 2006; Sabag-Daigle et al., 2015). Case et al. described the phenomenon of non-AHLs-producing microorganisms binding and utilizing AHLs produced by other organisms as eavesdropping (Case

\footnotetext{
Abbreviations: QS, quorum sensing; AHLs, N-acyl homoserine-lactones; $P$. aeruginosa, Pseudomonas aeruginosa; E. coli, Escherichia coli; qPCR, quantitative real-time PCR.
}

et al., 2008). Although SdiA can bind to DNA and regulate transcription in the absence of AHLs, the structural studies of SdiA suggest a double mode of action for AHLs on SdiA activity, by increasing both protein stability and DNA-binding affinity (Nguyen et al., 2015; Ishihama et al., 2016). Besides, a neighborjoining tree analysis revealed that SdiA of E. coli did not cluster with the LuxR homologs found in other enterobacterial species, but was closely related to the RhlR of $P$. aeruginosa (Gray and Garey, 2001).

Herein, we clarified the effect of QS on conjugation and investigated the underlying mechanisms by employing a mobilizable plasmid and E. coli-P. aeruginosa conjugation model. We found that QS signal molecules produced by P. aeruginosa inhibited interspecies conjugation by activating E. coli SdiA, resulting in decreased mRNA expression of traI in E. coli. Blockade of AHL-SdiA signaling using strains deficient in lasI, rhlI or sdiA significantly enhanced conjugative transfer. These findings provide new insight into the regulatory networks of conjugation, and offer novel potential targets for antibiotic resistance.

\section{MATERIALS AND METHODS}

\section{Bacterial Strains, Plasmids, and Growth Conditions}

The bacterial strains and plasmids used in this study are listed in Table 1. Bacteria were grown in Lysogeny Broth (LB) medium or on LB plates containing 1.5\% agar unless otherwise indicated. If required, antibiotics were added at the following final concentrations: ampicillin (Amp, $100 \mu \mathrm{g} / \mathrm{mL}$ ), gentamycin (Gm, $30 \mu \mathrm{g} / \mathrm{mL})$, chloramphenicol $(\mathrm{Cm}, 20 \mu \mathrm{g} / \mathrm{mL})$, kanamycin (Kan, $50 \mu \mathrm{g} / \mathrm{mL}$ ) and rifampicin (Rif, $50 \mu \mathrm{g} / \mathrm{mL}$ ).

\section{Growth Curves}

The indicated bacterial strains were cultured in LB overnight ( $8 \sim 10 \mathrm{~h}$ ) at $37^{\circ} \mathrm{C}$, then diluted to $0.5 \mathrm{MCF}$ (McFarland standard) and $3 \mathrm{~mL}$ cultures were grown at $37^{\circ} \mathrm{C}$ with shaking at $200 \mathrm{rpm}$. The samples were collected at the indicated time points and the $\mathrm{OD}_{600}$ values were determined.

\section{Plasmid Construction}

The plasmid pUCP24T was constructed by inserting the oriT fragment into pUCP24 (West et al., 1994), which contains a gene cassette $(a a c C 1)$ conferring gentamycin resistance in recipient cells. As a result, pUCP24T is not able to transfer on its own, but can transfer by using the conjugative apparatus of $E$. coli $S M 10 \lambda \pi$. Details of construction of the plasmids used to delete sdiA gene or express SdiA are described in the Supporting Materials and Methods.

\section{Construction of PAO1 lasl or rhll and E. coli $S M 10 \lambda \pi$ sdiA Deficient Mutants}

The phage $\lambda$ Red recombination system was employed for $s d i A$ deletion in E. coli $S M 10 \lambda \pi$, while the $s a c B$-based suicide vector system was adapted for knockout of lasI or rhlI in PAO1 (Zeng et al., 2016); further details are provided in the Supporting Materials and Methods. 
TABLE 1 | Bacterial strains and plasmids.

\begin{tabular}{|c|c|c|}
\hline Strains/plasmids & Genotype or characteristics & Source \\
\hline \multicolumn{3}{|l|}{ STRAINS } \\
\hline 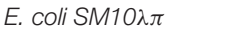 & thi thr leu tonA lacY supE recA::RP4-2-Tc::Mu Km $\lambda$ pir & Simon et al., 1983 \\
\hline E. coli $S M 10 \lambda \pi \Delta s d i A$ & 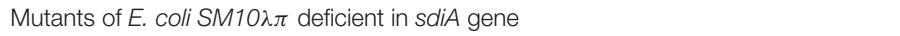 & This work \\
\hline E. coli EC600 & LacZ-, Nal' ${ }^{\mathrm{Rr}}, \mathrm{Rif}{ }^{\mathrm{R}}$ & Our lab \\
\hline E. coli BW25113 & $\Delta(\operatorname{araD}-\operatorname{araB}) 567, \Delta$ lacZ4787(::rrnB-3), lambda-, rph-1, $\Delta$ (rhaD-rhaB)568, hsdR514 & Our lab \\
\hline$P$. aeruginosa PAO1 & Wild-type & Stover et al., 2000 \\
\hline PAO1 Alasl & Mutants of PAO1 deficient in las/ gene & Our lab Zeng et al., 2016 \\
\hline PAO1 1 rhll & Mutants of PAO1 deficient in rhll gene & Our lab Zeng et al., 2016 \\
\hline \multicolumn{3}{|l|}{ PLASMIDS } \\
\hline pKD3 & oriR6K, FRT::cat::FRT template plasmid $\mathrm{Cm}^{R}, \mathrm{Amp}^{\mathrm{R}}$ & Datsenko and Wanner, 2000 \\
\hline pKD46 & oriR101 repA101ts P-araB-gam-bet-exo Amp ${ }^{R}$ & Datsenko and Wanner, 2000 \\
\hline pCP20 & pSC101 temperature-sensitive repliconts, $\mathrm{Flp}(\lambda \mathrm{Rp}), \mathrm{cl} 857, \mathrm{Cm}^{\mathrm{R}}, \mathrm{Amp}^{\mathrm{R}}$ & Datsenko and Wanner, 2000 \\
\hline pQF50 & Promoterless lacZ reporter plasmid, $\mathrm{Amp}^{\mathrm{R}}$ & Farinha and Kropinski, 1990 \\
\hline pQF50-tral & pQF50 derivative, containing tral promoter region, $\mathrm{Amp}^{R}$ & This work \\
\hline pUCP24T & 370 bp oriT fragment from pCVD442 cloned into pUCP24, ori1600, $\mathrm{Gm}^{\mathrm{R}}$ & Philippe et al., 2004 \\
\hline
\end{tabular}

$R i f^{R}, \mathrm{Km}^{R}, \mathrm{Cm}^{R}, \mathrm{Gm}^{R}$, and $\mathrm{Amp} \mathrm{p}^{R}$ stand for rifampicin, kanamycin, chloramphenicol, gentamycin and ampicillin resistance, respectively.

\section{Conjugation Experiments}

For the conjugation assays, the same amount $\left(0.5 \times 10^{7} \mathrm{CFU} / \mathrm{mL}\right.$, counted using the Sysmex UF-1000i ${ }^{\mathrm{TM}}$ Automated Urine Particle Analyzer; Tokyo, Japan) of mid-logarithmic phase donor (E. coli $S M 10 \lambda \pi$ harboring plasmid pUCP24T) and recipient cells (PAO1 or EC600) were mixed in $200 \mu \mathrm{L} \mathrm{LB}$ with or without the indicated HSLs in 96-well plates. After $6 \mathrm{~h}$ mating at $37^{\circ} \mathrm{C}$, the cultures were vigorously mixed and $30 \mu \mathrm{L}$ aliquots of each conjugation mixture were spread on LB agar containing $30 \mu \mathrm{g} / \mathrm{mL}$ Gm plus $100 \mu \mathrm{g} / \mathrm{mL}$ Amp for $S M 10 \lambda \pi-P A O 1$ or $30 \mu \mathrm{g} / \mathrm{mL}$ Gm plus 50 $\mu \mathrm{g} / \mathrm{mL}$ Rif for $S M 10 \lambda \pi-E C 600$ transconjugants. The numbers of transconjugant colonies were counted after overnight incubation at $37^{\circ} \mathrm{C}$.

\section{Quantification of HSLs by HPLc-MS/MS}

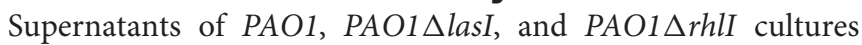
were collected for HPLC-MS/MS detection of HSLs; full details are provided in the Supporting Materials and Methods.

\section{$\beta$-Galactosidase Assays}

$\beta$-Galactosidase activities were performed on cells in the mid-log phase of growth according to the modified Miller's method (Giacomini et al., 1992). All tests were performed in triplicate.

\section{Electrophoretic Mobility Shift Assays (EMSA)}

His-SdiA fusion protein was expressed in E. coli BL21 (DE3) and purified via Ni-chelating affinity chromatography. Gel shift assays were carried out using the Lightshift Chemiluminescent EMSA kit according to the manufacturer's instructions (Thermo Scientific, Waltham, MA, USA), details are provided in the Supporting Materials and Methods.

\section{Real-Time PCR}

Total RNA was extracted using total RNA isolation reagent (Promega, Madison, WI, USA). Reverse transcription ( $1 \mu \mathrm{g}$ of total RNA) was performed with the PrimeScript RT reagent Kit (Takara, Dalian, Liaoning, China). The cDNA was subjected to qPCR on a ViiA ${ }^{\mathrm{TM}} 7$ Dx system (Applied Biosystems, Foster, CA, USA) using SYBR Green qPCR Master Mixes (ThermoFisher Scientific). The expression levels of the target genes were normalized to the expression of the internal control gene $(r p o D)$, using the $2^{-\Delta \Delta \mathrm{Ct}}$ method. The sequences of the primers are listed in Table S1.

\section{Statistical Analysis}

Data are expressed as the mean \pm standard error of the mean (SEM) of at least three independent experiments. The differences between groups were analyzed using the Student's $t$-test when two groups were compared or one-way ANOVA when more than two groups were compared. All analyses were performed using GraphPad Prism, version 5 (GraphPad Software, Inc., San Diego, CA, USA). All statistical tests were two-sided; $P<0.05$ was considered statistically significant.

\section{RESULTS}

\section{Deficiency of lasl or rhll in $P$. aeruginosa Promotes SM10 $\pi \pi-P A O 1$ Conjugation}

To elucidate the biological significance of the QS system in $P$. aeruginosa conjugation, we first constructed lasI or rhlI single gene-deficient mutants, named PAO1 LlasI and PAO1 $\Delta$ rhlI, respectively. In $P$. aeruginosa, lasI catalyzes the formation of 3-oxo-C12-HSL, which positively regulates the expression of RhlI. RhlI directs the synthesis of C4-HSL, which subsequently regulates pyocyanin production (O'Loughlin et al., 2013). In this study, despite the existence of $r h l I$ in the genome of PAO1 $\triangle$ lasI, both 3-oxo-C12-HSL and C4-HSL were barely detectable in the 
conditioned medium of this mutant strain using HPLC-MS/MS analysis. For PAO1 $\Delta$ rhll, the deficiency of rhlI in the genome led to an absence of C4-HSL in the conditioned medium of this mutant strain, whereas lasI and its product 3-oxo-C12-HSL were present at similar levels as the WT strain (Figure 1A and Figure $\mathrm{S} 1$ ). Furthermore, as a result of mutation of the QS system, both

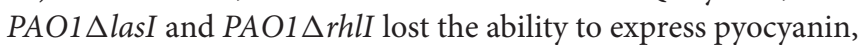
which could be rescued by exogenous addition of 3-oxo-C12HSL or C4-HSL (Figure 1B). Taken together, these results confirmed the successful creation of PAO1 strains deficient in lasI or rhli.

We subsequently examined the growth and conjugation

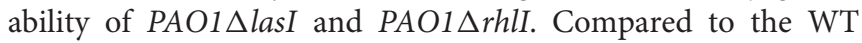
strain, deficiency of lasI or rhlI hardly affected the growth of PAO1 (Figure S2), but significantly promoted $S M 10 \lambda \pi-P A O 1$ conjugation (Figure 1C). Furthermore, exogenous 3-oxo-C12HSL or C4-HSL attenuated the interspecies conjugation ability

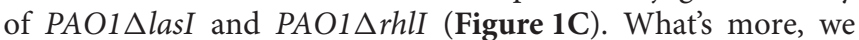
counted the amount of donor $S M 10 \lambda \pi$ after co-culture with

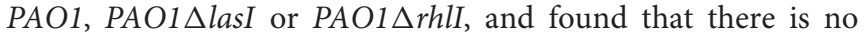
difference among the three groups (Figure S3), indicted that the observed effect of quorum sensing on conjugation efficiency was not due to the growth suppressive effect on $S M 10 \lambda \pi$. These data suggested that the QS system may negatively regulate $S M 10 \lambda \pi$ PAO1 conjugation.

\section{The Quorum Sensing System of $P$. aeruginosa Inhibits Conjugation by Activating SdiA of E. coli}

It is well recognized that AHLsq regulate gene transcription via binding to their receptor proteins (LuxR-like proteins). In this conjugation model, in contrast to the recipient cells PAO1, the donor E. coli $S M 10 \lambda \pi$ cells lack AHL synthase and therefore do not produce AHLs; however, these cells produce a LuxR homolog known as SdiA that can bind AHLs produced by other bacterial species to regulate gene transcription. Given that the conjugative apparatus exist in donor cells, we speculated that $P$. aeruginosa-released AHLs may act on SdiA of E. coli. To assess whether SdiA of E. coli is involved in the ability of $P$. aeruginosa's AHLs to inhibit $E$. coli- $P$. aeruginosa conjugation, we constructed the sdiA deficient mutant $S M 10 \lambda \pi \Delta s d i A$. As expected, deficiency of $s d i A$ in $S M 10 \lambda \pi$ significantly enhanced $E$. coli-P. aeruginosa conjugation, whereas overexpression of SdiA reversed the phenotype (Figure 2A). However, when using a AHLs-non-producing E. coli strain EC600 as the recipient cell, $S M 10 \lambda \pi \Delta s d i A$ did not increase conjugation ability compared to the WT strain (Figure 2B). More importantly, the conjugation efficiency of $S M 10 \lambda \pi$ and EC600 significantly decreased in the presence of exogenous 3-oxo-C12HSL and C4-HSL, while sdiA deletion in $S M 10 \lambda \pi$ abrogated the effects of AHLs on conjugation (Figure 2B), suggesting the inhibitory effect of SdiA on E. coli-P. aeruginosa or $S M 10 \lambda \pi$ EC600 conjugation is dependent on the presence of AHLs. In addition, growth curves demonstrated that deficiency of $s d i A$ in $E$. coli had no influence on cell proliferation (Figures S4, S5), confirming that the regulatory function of SdiA in conjugation in this model was not due to an altered growth rate.

Collectively, these data imply that AHLs produced by PAO1 may repress $S M 10 \lambda \pi-P A O 1$ conjugation through binding to SdiA of E. coli.

\section{The Interaction between $P$. aeruginosa HSL and $E$. coli SdiA Inhibits the Expression of tral in E. coli}

Mechanisms behind transcription regulation function of SdiA is being disclosed, it seems that genes with specific DNA sequences (SdiA-box) 5'-AAAAG(N8)GAAAA-3' in the promoter region may be the potential targets of SdiA (Yamamoto et al., 2001). In view of the presence of SdiA-box in the promoter of many SdiAregulated genes in our bioinformatics analysis (Table S2), we computationally mapped the DNA sequence in the RP4 plasmid to search for conjugation-related genes potentially regulated by SdiA. An SdiA-box sequence (5'-AAGAGcgattgagGAAAA-3') was identified -317 bp upstream of the traI start codon (Figure S6). Subsequently, EMSA assays confirmed the interaction between SdiA and the predicted SdiA-box of the traI promoter in vitro (Figures 3A,B). We therefore further evaluated the role of SdiA in the regulation of traI transcription. DNA fragments of traI promoter carrying the predicted SdiA-box was cloned upstream of the $\beta$-galactosidase gene in the pQF50-promoter reporter. When transformed into BW25113 (another $E$. coli strain without endogenous $\beta$-galactosidase compared to $S M 10 \lambda \pi$ ), the $\beta$-galactosidase activity of pQF50traI was greatly elevated, compared to that of the control, while addition of 3-oxo-C12-HSL and C4-HSL impaired this activity, which was severely attenuated when the sdiA was deleted (Figure 3B). Intriguing, when AHLs was absent, deletion of sdiA hardly affected $\beta$-galactosidase activity of pQF50-traI (Figure 3B), this is consistent with the phenotype shown in Figure 2. Compared with the WT strain, $S M 10 \lambda \pi \Delta s d i A$ showed higher mRNA expression of traI when cultured with PAO1 (Figure 3C). On the other hand, in the $S M 10 \lambda \pi-P A O 1$ co-culture system, deficiency of lasI or rhlI in PAO1 also led to enhanced expression of traI (Figure 3D), while supplementation with exogenous 3-oxo-C12-HSL and C4-HSL significantly repressed traI expression (Figure 3E). These results suggest that repressing traI expression in the donor cells may be a critical mechanism behind the inhibitory effect of the AHLs on conjugation.

In summary, we disclosed the cooperative effect of AHLs produced by recipient $P$. aeruginosa cells and SdiA of donor E. coli cells in the conjugation regulation. These findings indicate that QS may inhibit conjugation and prevent the excessive dissemination of plasmid.

\section{DISCUSSION}

Most recent publications in this field have focused on the regulatory function of QS in virulence and biofilm formation. Here, using E. coli (SM10 $\pi \pi)$ as donor cells and AHLs-producing $P$. aeruginosa (PAO1) or non-AHLs producing E. coli (EC600) as recipient cells, we identified a conjugation-inhibitory effect for 
A

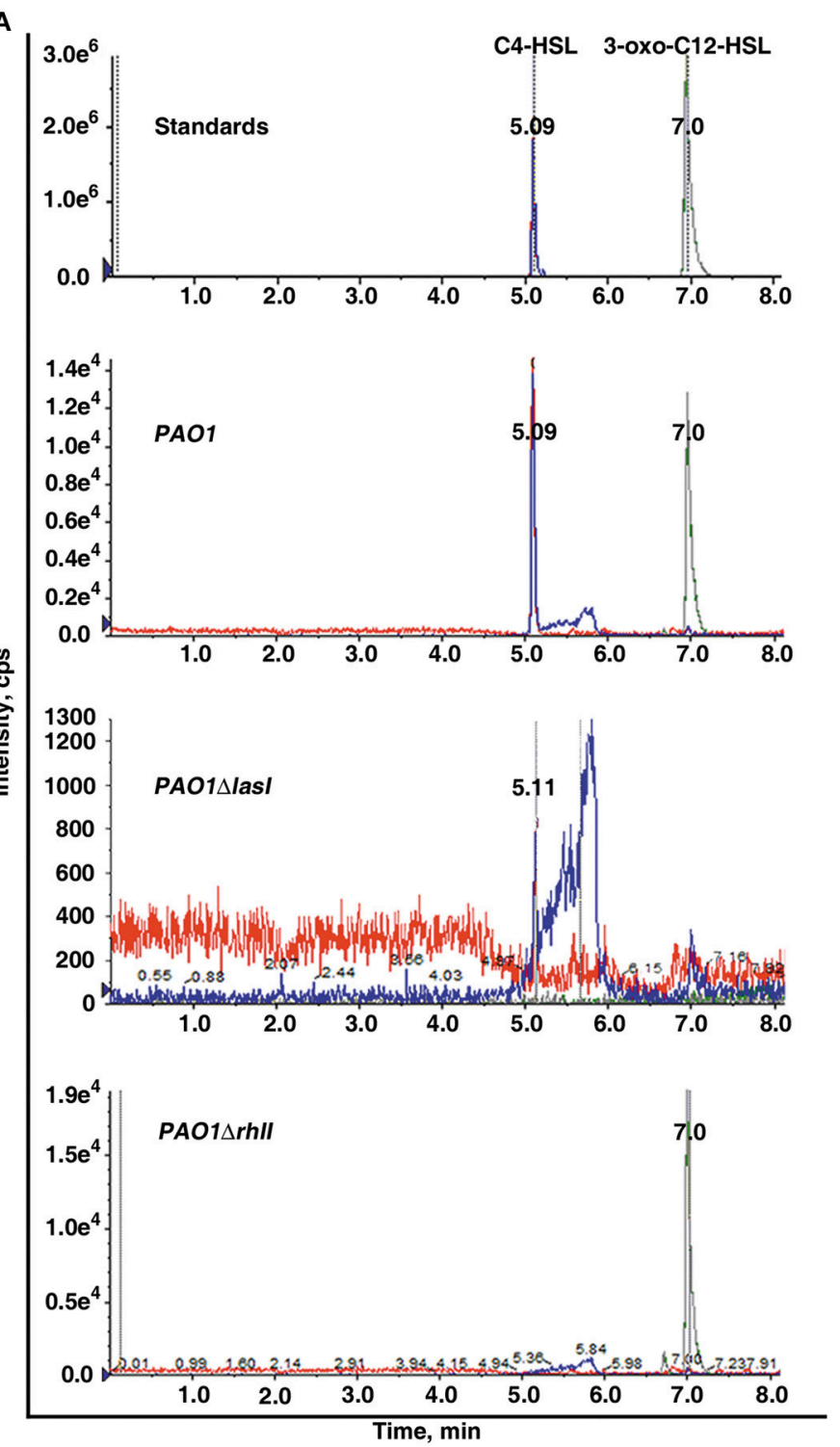

B

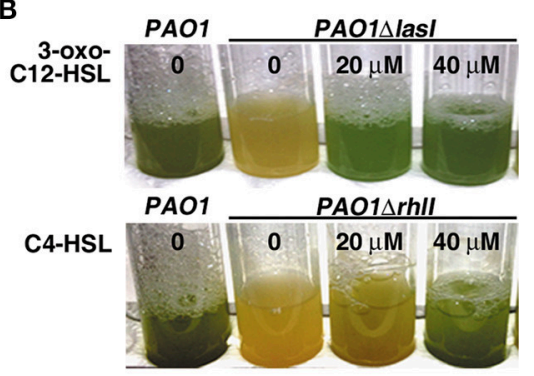

C
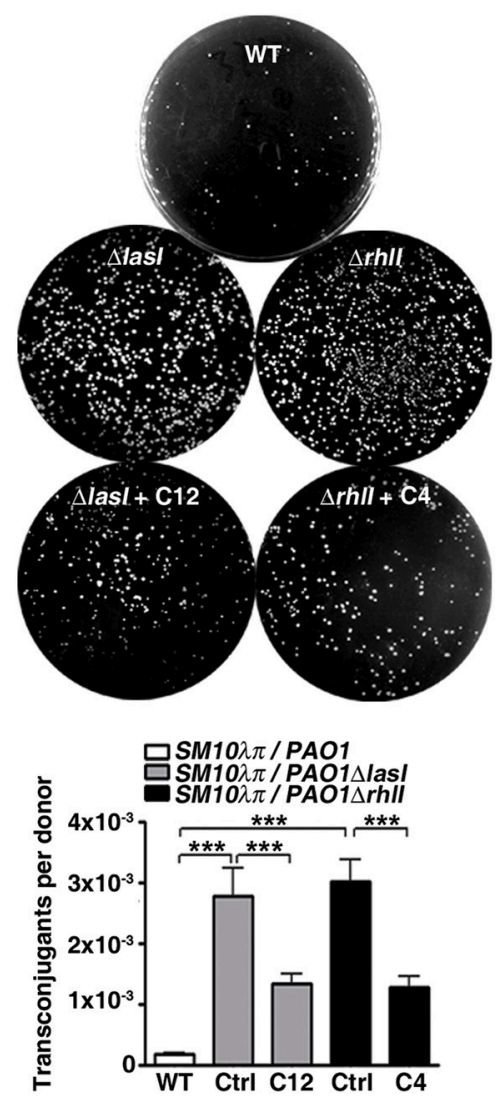

FIGURE 1 | The quorum sensing system of $\boldsymbol{P}$. aeruginosa inhibits conjugation between $\boldsymbol{P}$. aeruginosa and $\boldsymbol{E}$. coli. (A) Deficiency of the AHLs synthase genes rhll or las/ in P. aeruginosa (PAO1) resulted in the absence of C4-HSL or both 3-oxo-C12-HSL and C4-HSL, respectively. The 3-oxo-C12-HSL and C4-HSL in the cell-free supernatants were extracted with ethyl acetate and redissolved in methanol, followed by HPLC-MS/MS analysis. (B) Deficiency of las/ or rhll in $P$. aeruginosa abolished production of the downstream toxin of rhll system pyocyanin. PAO1, PAO1 $\triangle$ /as/ and PAO1 $\triangle$ rhll were cultured in the presence or absence of 3-oxo-C12-HSL or C4-HSL as indicated for $30 \mathrm{~h}$. (C) Deficiency of las/ or rhll in P. aeruginosa significantly promoted SM10 $\pi$-PAO1 conjugation; this effect could be

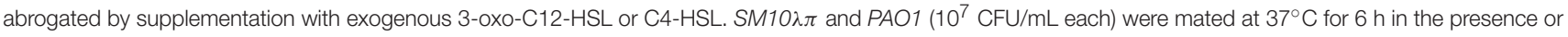
absence of $40 \mu \mathrm{M}$ of C4-HSL or 3-oxo-C12-HSL. Ctrl, control; C12, 3-oxo-C12-HSL; C4, C4-HSL. Values are mean \pm SEM of at least three independent experiments; ${ }^{* \star *} P<0.001$.

QS based on the following evidence. First, for $S M 10 \lambda \pi$ and $P A O 1$ co-culture system in which AHLs is normally self-sustained, deficiency of the AHLs-producing genes lasI or rhlI in PAO1 or the solo AHLs receptor SdiA in $S M 10 \lambda \pi$ promoted $S M 10 \lambda \pi$ $P A O 1$ conjugation, while supplementation with exogenous 3oxo-C12-HSL or C4-HSL abrogated the enhanced conjugation

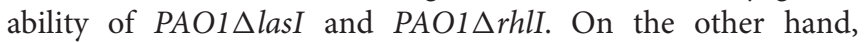
for both non-AHLs producing $S M 10 \lambda \pi$ and EC600 mixed cultures, stimulation with exogenous 3-oxo-C12-HSL and C4HSL inhibited conjugation, while deletion of $s d i A$ in $S M 10 \lambda \pi$ attenuated this effect. Conventionally, conjugation is considered to be mainly regulated by the self-transmissible plasmids. While our results indicate that QS system of donor and recipient cells may play a role in conjugation regulation.

Conjugation enables the dissemination of virulence genes and antibiotic resistance genes, which leads to the adaption of bacteria 


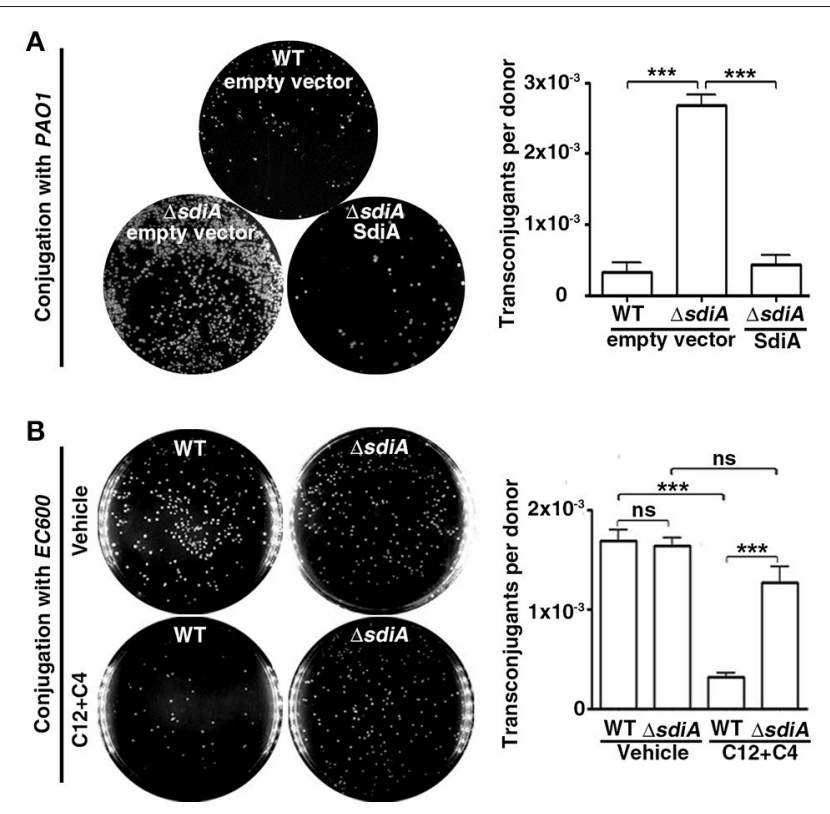

FIGURE 2 | AHL inhibits SM10 $\pi$-PAO1 conjugation via a mechanism dependent on SdiA of E. coli. (A) Deficiency of $s d i A$ in E. coli significantly promoted $S M 10 \lambda \pi-P A O 1$ conjugation. E. coli $S M 10 \lambda \pi$ and $P$. aeruginosa PAO1 (10 $\mathrm{CFU} / \mathrm{mL}$ each) were mated at $37^{\circ} \mathrm{C}$ for $6 \mathrm{~h}$. (B) Deficiency of sdiA in $S M 10 \lambda \pi$ did not significantly affect $S M 10 \lambda \pi-E C 600$ conjugation, but could rescue 3-oxo-C12-HSL and C4-HSL (C4/12)-inhibited conjugation. SM10 $\lambda \pi$ was cultured in the presence of DMSO or $40 \mu \mathrm{M}$ C4-HSL and 3-oxo-C12-HSL for $6 \mathrm{~h}$, followed by conjugation with EC600. Values are mean $\pm \mathrm{SEM}$ of at least three independent experiments; ns, not significant, ${ }^{\star \star *} P<0.001$.

to new circumstances (Norman et al., 2009). Therefore, the ability to inhibit conjugation may be a potentially efficacious strategy for avoiding the spread of resistance traits. Here, we demonstrate that AHL-SdiA is capable of suppressing conjugation. Most SdiAexpression bacteria, such as Escherichia, Salmonella and Shigella are enterobacteria, while many biological evidences suggest a lack of HSLs in the normal mammalian intestine (Swearingen et al., 2013), despite the presence of AHLs in bovine rumen (Hughes et al., 2010). Thus, although $P$. aeruginosa could be detected in stool sample in our clinical microbiology laboratory, future studies are needed to illuminate the role of AHL-SdiA signaling in pathogenic bacteria communities within the gastrointestinal tract.

To date, many SdiA regulon members have been described (Kanamaru et al., 2000; Wei et al., 2001; Dyszel et al., 2010; Sabag-Daigle et al., 2015). Here we report the identification of SdiA-regulated and AHL-responsive gene traI in the plasmid RP4. TraI is reported to function as a relaxase enzyme that creates a nick at the oriT of conjugative plasmids, which is required to initiate conjugation (Furuya and Komano, 2000). We discovered a DNA motif recognized by SdiA in the promoter region of the traI gene in the plasmid RP4, and the interaction between SdiA and the predicted SdiA-box was validated in vitro using an EMSA. However, some SdiA-regulated genes do not have this particular SdiA-box (Dyszel et al., 2010; Swearingen et al., 2013; Abed et al., 2014; Nguyen et al., 2015), there
A

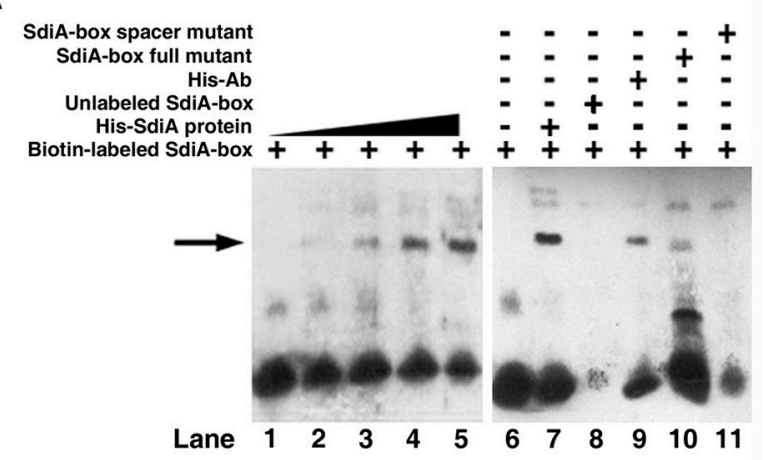

B

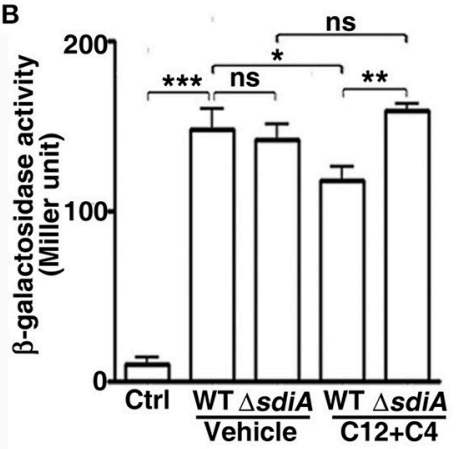

C

E
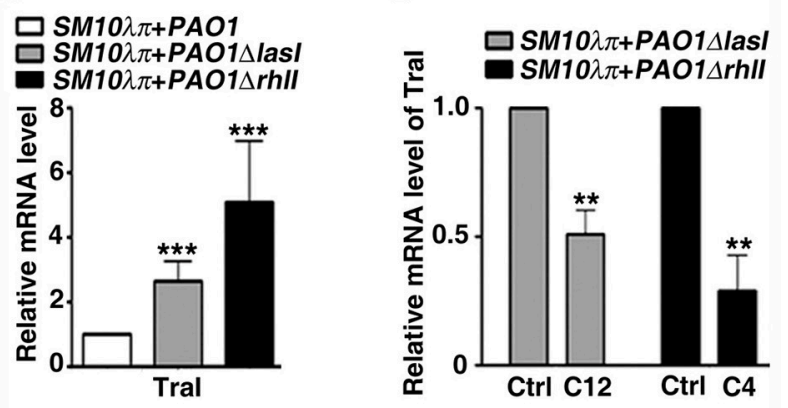

FIGURE 3 | The AHL-SdiA interaction represses the expression of Tral in $\boldsymbol{E}$. coli. (A) EMSA verified the interaction of SdiA protein with the putative SdiA-box of the tral promoter. Biotin-labeled SdiA-box (50 fmol) was added to each reaction with $0,2,4,8$, or 16 pmol $E$. coli His-SdiA fusion protein (lane 1 to lane 5 , respectively). The specificity analysis (lane 6 to lane 11) was performed in the presence (-) or absence $(+)$ of His-SdiA fusion protein (8 pmol) and biotin-labeled SdiA-box (50 fmol, lanes 7), 200-fold unlabeled SdiA-box (lanes 8), anti-His antibody (lanes 9), or 200-fold SdiA-box full or spacer mutant competitors (lane 10, 11). (B) Activity of $\beta$-galactosidase reporters containing the predicted SdiA-box in genomic regions upstream of tral under various conditions. E.coli BW25113 or E.coli BW25113 $\Delta$ sdiA were cultured with SM10 $\lambda \pi$ in the presence of DMSO (Ctrl) or $40 \mu \mathrm{M} \mathrm{C4-HSL}$ and 3-oxo-C12-HSL for $6 \mathrm{~h}$, followed by $\beta$-galactosidase activity analysis. (C) Deficiency of sdiA promoted tral expression in E. coli. (D) Deficiency of lasl/rhll promoted tral expression in E. coli. For (C,D), $10^{7} \mathrm{CFU} / \mathrm{mL}$ (each) of the indicated $E$. coli donor and recipient $P$. aeruginosa cells were mated at $37^{\circ} \mathrm{C}$ for $6 \mathrm{~h}$, followed by real-time PCR analysis. The rpoD gene of $E$. coli was used as an internal control. (E) Exogenous 3-oxo-C12-HSL or C4-HSL inhibited tral expression in E. coli. PAO1 $\Delta$ lasl or PAO1 $\Delta r h l l$ were cultured with $S M 10 \lambda \pi$ in the presence of DMSO (Ctrl) or $40 \mu \mathrm{M}$ C4-HSL and 3-oxo-C12-HSL for $6 \mathrm{~h}$, followed by real-time PCR analysis. The rpoD gene of $E$. coli was used as an internal control. Ctrl, control; C12, 3-oxo-C12-HSL; C4, C4-HSL. Values are mean \pm SEM of at least three independent experiments; ${ }^{*} P<0.05$; ${ }^{\star \star} P<$ $0.01 ;{ }^{\star * \star} P<0.001$. 
may be other conjugation-related genes repressed by AHL-SdiA. Moreover, the EMSA was performed without addition of AHLs, so it seems that high concentration of SdiA could bind to traI promoter in the absence of AHLs in vitro (Figure 3A), however, the reporter system (Figure 3B) and conjugation experiment (Figure 2B) showed that in the absence of AHLs, deletion of sdiA hardly affected the promoter activity of traI, as well as conjugation frequency in vivo. Thus, we proposed AHLs may increase both SdiA protein stability and traI promoter-binding affinity to repress traI expression.

Despite the advantages of conjugation for bacteria, the introduction of novel genes into the pre-existing, well-tuned genetic background is a source of genetic conflict, and possession of the conjugation-associated machinery also places a burden on the host arising from the energy expended to create and maintain the conjugative apparatus and its associated features (Zatyka and Thomas, 1998; Baltrus, 2013; San Millan et al., 2015). This raises the question of how host bacteria minimize the metabolic cost while obtaining the benefits provided by conjugation. In this study, we found that under normal conditions, when mobilizable plasmid containing a resistance gene was not required by PAO1 (Table S3), conjugation between $S M 10 \lambda \pi$ and PAO1 was inhibited via the LasI/RhlI-HSL-SdiA pathway. These findings reveal that QS system may play a role in protecting host cells against external conjugative plasmids.

Utilizing ecological data from 2801 samples, Freilich et al. explored the ubiquitous competitive and cooperative interactions between the bacteria within natural communities (Freilich et al., 2011). Nonetheless, revealing more detail of the strategies bacteria adopt for survival in mixed cultures remains a major challenge. The E. coli-P. aeruginosa conjugation model has been widely used in studies of bacterial conjugation, and the most prevalent donor strain is E. coli $S M \lambda \pi$ in which the RP4 plasmid is chromosomally-integrated. Thus, conjugation-associated genes, such as traI initially only exist in and are expressed by the E. coli $(S M 10 \lambda \pi)$ cells, similarly rhlI and lasI are only expressed by PAO1. This makes it easy to detect the expression of these genes in $E$. coli $(S M 10 \lambda \pi)$ and PAO1, specifically in mixed-cultures. Using this co-culture system, we found that LasI/RhlI and SdiA jointly repressed traI expression in $E$. coli and inhibited

\section{REFERENCES}

Abed, N., Grépinet, O., Canepa, S., Hurtado-Escobar, G. A., Guichard, N., Wiedemann, A., et al. (2014). Direct regulation of the pefI-srgC operon encoding the Rck invasin by the quorum-sensing regulator SdiA in Salmonella Typhimurium. Mol. Microbiol. 94, 254-271. doi: 10.1111/mmi.12738

Arthur, D. C., Edwards, R. A., Tsutakawa, S., Tainer, J. A., Frost, L. S., and Glover, J. N. M. (2011). Mapping interactions between the RNA chaperone FinO and its RNA targets. Nucleic Acids Res. 39, 4450-4463. doi: 10.1093/nar/gkr025

Baltrus, D. A. (2013). Exploring the costs of horizontal gene transfer. Trends Ecol. Evol. 28, 489-495. doi: 10.1016/j.tree.2013.04.002

Bassler, B. L., and Losick, R. (2006). Bacterially speaking. Cell 125, 237-246. doi: 10.1016/j.cell.2006.04.001

Bleves, S., Soscia, C., Nogueira-Orlandi, P., Lazdunski, A., and Filloux, A. (2005). Quorum sensing negatively controls type III secretion regulon expression in Pseudomonas aeruginosa PAO1. J. Bacteriol. 187, 3898-3902. doi: 10.1128/JB.187.11.3898-3902.2005
$S M 10 \lambda \pi-P A O 1$ conjugation, indicating that the QS system may provide a mechanism of cooperative regulation between bacteria.

In conclusion, the findings of this study highlight the regulatory role for the QS system in conjugation, and expand our understanding of the bacterial communication and defense systems of $P$. aeruginosa.

\section{AUTHOR CONTRIBUTIONS}

YL, JZ, XH, BH, and CC designed research; YL, JZ, BW, RC, and NZ performed research; SE and YQL contributed new reagents/analytic tools; YL, JZ, and BW analyzed data; YL, JZ, and CC wrote the paper.

\section{FUNDING}

This work was supported by the National Natural Science Foundation of China (Grant No. 81572058, 81672081), the Natural Science Foundation of Guangdong Province (Grant No. 2014A030313143) and the Science and Technology Planning Project of Guangdong Province (Grant No. 2016A020215236).

\section{ACKNOWLEDGMENTS}

We are very grateful to Prof. B.L. Wanner (Department of Biological Sciences, Purdue University, West Lafayette, USA) for generously providing the $\lambda$ Red recombination system, Prof. E. Peter Greenberg (Department of Microbiology, University of Washington School of Medicine, Seattle, Washington, USA) for providing pQF50 plasmid and Prof. H. P. Schweizer (Department of Microbiology, Immunology and Pathology, College of Veterinary Medicine and Biological Sciences, Colorado State University, Fort Collins, Colorado, USA) for providing the plasmid pUCP24.

\section{SUPPLEMENTARY MATERIAL}

The Supplementary Material for this article can be found online at: http://journal.frontiersin.org/article/10.3389/fcimb. 2017.00007/full\#supplementary-material

Carballeira, J. D., González-Pérez, B., Moncalián, G., and De La Cruz, F. (2014). A high security double lock and key mechanism in HUH relaxases controls oriT-processing for plasmid conjugation. Nucleic Acids Res. 42, 10632-10643. doi: 10.1093/nar/gku741

Case, R. J., Labbate, M., and Kjelleberg, S. (2008). AHL-driven quorum-sensing circuits: their frequency and function among the Proteobacteria. ISME J. 2, 345-349. doi: 10.1038/ismej.2008.13

Datsenko, K. A., and Wanner, B. L. (2000). One-step inactivation of chromosomal genes in Escherichia coli K-12 using PCR products. Proc. Natl. Acad. Sci. U.S.A. 97, 6640-6645. doi: 10.1073/pnas.120163297

Dyszel, J. L., Soares, J. A., Swearingen, M. C., Lindsay, A., Smith, J. N., and Ahmer, B. M. M. (2010). E. coli K-12 and EHEC genes regulated by SdiA. PLoS ONE 5:e8946. doi: 10.1371/journal.pone.0008946

Farinha, M. A., and Kropinski, A. M. (1990). Construction of broadhost-range plasmid vectors for easy visible selection and analysis of promoters. J. Bacteriol. 172, 3496-3499. doi: 10.1128/jb.172.6.3496-3499. 1990 
Fernández-López, C., Bravo, A., Ruiz-Cruz, S., Solano-Collado, V., Garsin, D. A., Lorenzo-Díaz, F., et al. (2014). Mobilizable rolling-circle replicating plasmids from gram-positive bacteria: a low-cost conjugative transfer. Microbiol. Spectr. 2, 1-33. doi: 10.1128/microbiolspec.PLAS-0008-2013

Freilich, S., Zarecki, R., Eilam, O., Segal, E. S., Henry, C. S., Kupiec, M., et al. (2011). Competitive and cooperative metabolic interactions in bacterial communities. Nat. Commun. 2, 589-595. doi: 10.1038/ncomms 1597

Furuya, N., and Komano, T. (2000). Initiation and termination of DNA transfer during conjugation of IncI1 plasmid R64: roles of two sets of inverted repeat sequences within oriT in termination of R64 transfer. J. Bacteriol. 182, 3191-3196. doi: 10.1128/JB.182.11.3191-3196.2000

Gambello, M. J., Kaye, S., and Iglewski, B. H. (1993). LasR of Pseudomonas aeruginos $a$ is a transcriptional activator of the alkaline protease gene (apr) and an enhancer of exotoxin A expression. Infect. Immun. 61, 1180-1184.

García-Contreras, R. (2016). Is quorum sensing interference a viable alternative to treat Pseudomonas aeruginosa infections? Front. Microbiol. 7:1454. doi: 10.3389/fmicb.2016.01454

Giacomini, A., Corich, V., Ollero, F. J., Squartini, A., and Nuti, M. P. (1992). Experimental conditions may affect reproducibility of the beta-galactosidase assay. FEMS Microbiol. Lett. 100, 87-90. doi: 10.1111/j.1574-6968.1992.tb05687.x

Gray, K. M., and Garey, J. R. (2001). The evolution of bacterial LuxI and LuxR quorum sensing regulators. Microbiology 147, 2379-2387. doi: 10.1099/00221287-147-8-2379

Hassett, D. J., Ma, J. F., Elkins, J. G., McDermott, T. R., Ochsner, U. A., West, S. E., et al. (1999). Quorum sensing in Pseudomonas aeruginosa controls expression of catalase and superoxide dismutase genes and mediates biofilm susceptibility to hydrogen peroxide. Mol. Microbiol. 34, 1082-1093. doi: 10.1046/j.1365-2958.1999.01672.x

Hughes, D. T., Terekhova, D. A., Liou, L., Hovde, C. J., Sahl, J. W., Patankar, A. V., et al. (2010). Chemical sensing in mammalian hostbacterial commensal associations. Proc. Natl. Acad. Sci. U.S.A. 107, 9831-9836. doi: 10.1073/pnas.1002551107

Ishihama, A., Shimada, T., and Yamazaki, Y. (2016). Transcription profile of Escherichia coli: genomic SELEX search for regulatory targets of transcription factors. Nucleic Acids Res. 44, 2058-2074. doi: 10.1093/nar/gkw051

Jimenez, P. N., Koch, G., Thompson, J. A., Xavier, K. B., Cool, R. H., and Quax, W. J. (2012). The multiple signaling systems regulating virulence in Pseudomonas aeruginosa. Microbiol. Mol. Biol. Rev. 76, 46-65. doi: 10.1128/MMBR.05007-11

Jones, S., Yu, B., Bainton, N. J., Birdsall, M., Bycroft, B. W., Chhabra, S. R., et al. (1993). The lux autoinducer regulates the production of exoenzyme virulence determinants in Erwinia carotovora and Pseudomonas aeruginosa. EMBO J. 12, 2477-2482.

Kanamaru, K., Tatsuno, I., Tobe, T., and Sasakawa, C. (2000). SdiA, an Escherichia coli homologue of quorum-sensing regulators, controls the expression of virulence factors in enterohaemorrhagic Escherichia coli O157:H7. Mol. Microbiol. 38, 805-816. doi: 10.1046/j.1365-2958.2000.02171.x

Nguyen, Y., Nguyen, N. X., Rogers, J. L., Liao, J., MacMillan, J. B., Jiang, Y., et al. (2015). Structural and mechanistic roles of novel chemical ligands on the SdiA quorum-sensing transcription regulator. MBio 6, 2092-2096. doi: $10.1128 / \mathrm{mBio} .02429-14$

Norman, A., Hansen, L. H., and Sørensen, S. J. (2009). Conjugative plasmids: vessels of the communal gene pool. Philos. Trans. R. Soc. Lond. B. Biol. Sci. 364, 2275-2289. doi: 10.1098/rstb.2009.0037

O’Loughlin, C. T., Miller, L. C., Siryaporn, A., Drescher, K., Semmelhack, M. F., and Bassler, B. L. (2013). A quorum-sensing inhibitor blocks Pseudomonas aeruginosa virulence and biofilm formation. Proc. Natl. Acad. Sci. U.S.A. 110, 17981-17986. doi: 10.1073/pnas.1316981110

Passador, L., Cook, J. M., Gambello, M. J., Rust, L., and Iglewski, B. H. (1993). Expression of Pseudomonas aeruginosa virulence genes requires cell-to-cell communication. Science 260, 1127-1130. doi: 10.1126/science. 8493556

Pearson, J. P., Gray, K. M., Passador, L., Tucker, K. D., Eberhard, A., Iglewski, B. H., et al. (1994). Structure of the autoinducer required for expression of Pseudomonas aeruginosa virulence genes. Proc. Natl. Acad. Sci. U.S.A. 91, 197-201. doi: 10.1073/pnas.91.1.197

Philippe, N., Alcaraz, J. P., Coursange, E., Geiselmann, J., and Schneider, D. (2004). Improvement of pCVD442, a suicide plasmid for gene allele exchange in bacteria. Plasmid 51, 246-255. doi: 10.1016/j.plasmid.2004.02.003
Sabag-Daigle, A., Dyszel, J. L., Gonzalez, J. F., Ali, M. M., and Ahmer, B. M. M. (2015). Identification of sdiA-regulated genes in a mouse commensal strain of Enterobacter cloacae. Front. Cell. Infect. Microbiol. 5:47. doi: 10.3389/fcimb.2015.00047

San Millan, A., Toll-Riera, M., Qi, Q., and MacLean, R. C. (2015). Interactions between horizontally acquired genes create a fitness cost in Pseudomonas aeruginosa. Nat. Commun. 6, 6845-6852. doi: 10.1038/ncomms7845

Simon, R., Priefer, U., and Pühler, A. (1983). A broad host range mobilization system for in vivo genetic engineering: transposon mutagenesis in gram negative bacteria. Nat. Biotechnol. 9, 784-791. doi: 10.1038/nbt1183-784

Smith, J. L., Fratamico, P. M., and Yan, X. (2011). Eavesdropping by bacteria: the role of SdiA in Escherichia coli and Salmonella enterica serovar typhimurium quorum sensing. Foodborne Pathog. Dis. 8, 169-178. doi: $10.1089 /$ fpd.2010.0651

Smith, J. N., and Ahmer, B. M. M. (2003). Detection of other microbial species by Salmonella: expression of the SdiA regulon. J. Bacteriol. 185, 1357-1366. doi: 10.1128/JB.185.4.1357-1366.2003

Stover, C. K., Pham, X. Q., Erwin, A. L., Mizoguchi, S. D., Warrener, P., Hickey, M. J., et al. (2000). Complete genome sequence of Pseudomonas aeruginosa PAO1, an opportunistic pathogen. Nature 406, 959-964. doi: 10.1038/35023079

Swearingen, M. C., Sabag-Daigle, A., and Ahmer, B. M. M. (2013). Are there acylhomoserine lactones within mammalian intestines? J. Bacteriol. 195, 173-179. doi: 10.1128/JB.01341-12

Toder, D. S., Gambello, M. J., and Iglewski, B. H. (1991). Pseudomonas aeruginosa LasA: a second elastase under the transcriptional control of lasR. Mol. Microbiol. 5, 2003-2010. doi: 10.1111/j.1365-2958.1991.tb00822.x

Wagner, V. E., Bushnell, D., Passador, L., Brooks, A. I., and Iglewski, B. H. (2003). Microarray analysis of Pseudomonas aeruginosa quorum-sensing regulons: effects of growth phase and environment. J. Bacteriol. 185, 2080-2095. doi: 10.1128/JB.185.7.2080-2095.2003

Wei, Y., Lee, J. M., Smulski, D. R., and LaRossa, R. A. (2001). Global impact of sdiA amplification revealed by comprehensive gene expression profiling of Escherichia coli. J. Bacteriol. 183, 2265-2272. doi: 10.1128/JB.183.7.2265-2272.2001

West, S. E., Schweizer, H. P., Dall, C., Sample, A. K., and RunyenJanecky, L. J. (1994). Construction of improved Escherichia-Pseudomonas shuttle vectors derived from $\mathrm{pUC18/19}$ and sequence of the region required for their replication in Pseudomonas aeruginosa. Gene 148, 81-86. doi: 10.1016/0378-1119(94)90237-2

Whiteley, M., Lee, K. M., and Greenberg, E. P. (1999). Identification of genes controlled by quorum sensing in Pseudomonas aeruginosa. Proc. Natl. Acad. Sci. U.S.A. 96, 13904-13909. doi: 10.1073/pnas.96.24.13904

Yamamoto, K., Yata, K., Fujita, N., and Ishihama, A. (2001). Novel mode of transcription regulation by SdiA, an Escherichia coli homologue of the quorum-sensing regulator. Mol. Microbiol. 41, 1187-1198. doi: 10.1046/j.1365-2958.2001.02585.x

Yao, Y., Martinez-Yamout, M. A., Dickerson, T. J., Brogan, A. P., Wright, P. E., and Dyson, H. J. (2006). Structure of the Escherichia coli quorum sensing protein SdiA: activation of the folding switch by acyl homoserine lactones. J. Mol. Biol. 355, 262-273. doi: 10.1016/j.jmb.2005.10.041

Zatyka, M., and Thomas, C. M. (1998). Control of genes for conjugative transfer of plasmids and other mobile elements. FEMS Microbiol. Rev. 21, 291-319. doi: 10.1111/j.1574-6976.1998.tb00355.x

Zeng, J., Zhang, N., Huang, B., Cai, R., Wu, B., E, S., et al. (2016). Mechanism of azithromycin inhibition of HSL synthesis in Pseudomonas aeruginosa. Sci. Rep. 6:24299. doi: $10.1038 /$ srep24299

Conflict of Interest Statement: The authors declare that the research was conducted in the absence of any commercial or financial relationships that could be construed as a potential conflict of interest.

Copyright $\odot 2017$ Lu, Zeng, Wu, E, Wang, Cai, Zhang, Li, Huang, Huang and Chen. This is an open-access article distributed under the terms of the Creative Commons Attribution License (CC BY). The use, distribution or reproduction in other forums is permitted, provided the original author(s) or licensor are credited and that the original publication in this journal is cited, in accordance with accepted academic practice. No use, distribution or reproduction is permitted which does not comply with these terms. 